\title{
Developments in the meiotic analysis of hybrids. IV. Utilizing datasets with merged figure classes
}

\author{
C. G. D. CHAPMAN \& G. KIMBER* \\ PBI Cambridge, Maris Lane, Trumpington, Cambridge CB2 2LQ, U.K. and *Department of Agronomy, Curtis Hall, \\ University of Missouri-Columbia, Columbia, MO 65211, U.S.A.
}

\begin{abstract}
The meiotic analysis of hybrids generally requires that the various classes of meiotic figures are scored separately. Many reported data, however, merge the numbers of rod and ring bivalents and open and closed quadrivalents together. Amongst other things, this prevents a direct assessment of $c$, the mean arm-pairing frequency. An approach is described which optimizes simultaneously for both $c$ and $x$, the measure of relative affinity, and which should circumvent this problem. In practice, however, only the merging of quadrivalent classes in tetraploid and pentaploid hybrids can be dealt with successfully. Estimates of $x$, and the choice of best fit model are highly unreliable in all other cases where rod and ring bivalent classes have also been merged. The full description of these figure classses is critical to meiotic analysis.
\end{abstract}

Keywords: meiotic analysis, merged figure classes.

\section{Introduction}

The analysis of the meiotic figures seen at metaphase I in species hybrids is a powereful tool for investigating genomic and taxonomic relationships in plants. The use of mathematical models of chromosome pairing to analyse the patterns of association gives a more objective assessment of relationships than does simple inspection. In this series of papers (Chapman \& Kimber 1992a-c) amended approaches to the analysis of triploid, tetraploid and pentaploid hybrids have been developed that better reflect present theory and assumptions about the process of chromosome pairing, and a modified approach to optimizing for $x$, the measure of relative affinity, incorporated.

One practical shortcoming of these analyses is that they require the observed data to which they are applied to categorize fully all the classes of meiotic figures. In practice many datasets reported in the literature merge some figure classes together. Most commonly closed and open quadrivalents are combined in one class of quadrivalents, and frequently rod and ring bivalents will also be merged. Kimber \& Pignone (1982) developed an approach to deal with merged bivalent classes in triploid hybrids first by estimating $c$, the mean arm-pairing frequency, from the number of univalents and bivalents, then in turn using this to estimate those proportions of the bivalents that were rods and rings. Subsequently the meiotic analysis of Alonso \& Kimber (1981) could be applied. In the case of higher ploidy hybrids with more complex pairing patterns, however, such direct estimates cannot be made. This paper descibes and assesses the value of a more general approach which may be applied to any level of ploidy. The terms pairing, meiotic figure, dataset, meiotic analysis and $c$ are used as discussed by Chapman \& Kimber (1992a).

\section{Theory}

The merging of bivalent and/or quadrivalent figure classes creates two difficulties in meiotic analysis. The first is that in the process of optimization observed and calculated figure numbers for given values of $c$ and $x$ are compared by calculating the weighted sums of squares of difference (WSSD) (Chapman \& Kimber, 1992a) for open and closed figures separately. This problem may be dealt with by adding the calculated numbers of the appropriate open and closed figures to produce figure classes that match those in the observed dataset. The WSSD is then calculated on these merged classes.

The second problem is that it is no longer possible to determine $c$ by direct calculation. A bivalent may have one or two bound arms, depending on whether it is a rod or ring. Similarly a quadrivalent has three 
bound arms if it is open, or four if it is closed. However, maximum and minimum possible values for $c$ may be calculated first by assuming all bivalents and quadrivalents are closed, then that they are all open. It is then possible to search simultaneously for values of $c$ within these limits and $x$ within its permitted range (0.5-1.0) for that combination that returns the minimum WSSD between the observed and calculated figure numbers.

The computer programs previously developed for triploid, tetraploid and pentaploid hybrids have been adapted for this purpose by merging the relevant calculated figure classes, and the inclusion of a twodimensional search routine which optimizes for both $x$ and $c$. They are written in TURBO PASCAL to run on the Apple Macintosh.

\section{Methods}

This approach to dealing with merged figure classes was tested on the same datasets as those examined by Alonso \& Kimber (1981), Kimber \& Alonso (1981) and Espinasse \& Kimber (1981) for triploid, tetraploid and pentaploid hybrids respectively. Each complete dataset was first analysed according to Chapman \& Kimber $(1992 \mathrm{a}-\mathrm{c})$. For the triploid hybrids the rod and ring bivalents were then added together and analysed by the procedures outlined above. The approach of Kimber \& Pignone (1982) was also tested by adding together rod ring bivalents into one class, estimating rod and ring numbers according to their formulae, then analysing the modified data after Chapman \& Kimber (1992a).

For the tetraploid and the pentaploid hybrids, the open and closed quadrivalent classes were first merged and the datasets analysed as set out in the theory section. Then the rod and ring bivalents were also merged and, again, the datasets analysed as above.

\section{Results and discussion}

Table 1 compares the analyses of the original and modified datasets by regression. For the tetraploid and pentaploid hybrids where there are three $(2: 2,2: 1: 1$ and $3: 1)$ and five $(2: 2: 1,3: 2,2: 1: 1: 1,3: 1: 1$ and $4: 1)$ possible models, respectively, the values of $c$ and $x$ being compared are those for the best fit model as determined from analysis of the complete dataset.

In the cases where quadrivalent classes have been merged in the tetraploids and pentaploids, the estimate of $c$ is very close to that calculated from the original data with the fitted regression lines having an intercept near zero, a slope very close to one, and a high correlation coefficient. The estimates of $c$ where bivalent classes have been merged in the triploid hybrids, or both bivalents and quadrivalents are merged in the tetraploids and pentaploids are less satisfactory but still correspond well with the calculated values.

For the tetraploids and the pentaploids, estimates of $x$ for the best fit model are satisfactory where only the quadrivalent classes are merged. For tetraploids where the bivalent and quadrivalents merged the estimate of $x$ is reasonable but fortriploids and pentaploids with both bivalents and quadrivalents merged the estimates are poor. Furthermore, the best fit choice amongst the three models for the tetraploids and five models for the pentaploids, when bivalent and quadrivalent classes are merged is highly unreliable, coinciding with that from the full dataset 14 out of 19 cases for the tetraploid and only 7 out of 16 cases for the pentaploid.

Both approaches to the analysis of triploid data with merged bivalent classes yield similar estimates of $x$, with a clear overestimation of $x$ at values below about $x=0.9$. Apparently the tendency of both methods is to overestimate $c$ slightly and hence the numbers of ring bivalents. In consequence the estimate of $x$, which

Table 1 Comparisons of $c$ and $x$ from full $\left(c_{f}\right.$ and $\left.x_{f}\right)$ versus merged data $\left(c_{m}\right.$ and $\left.x_{m}\right)$

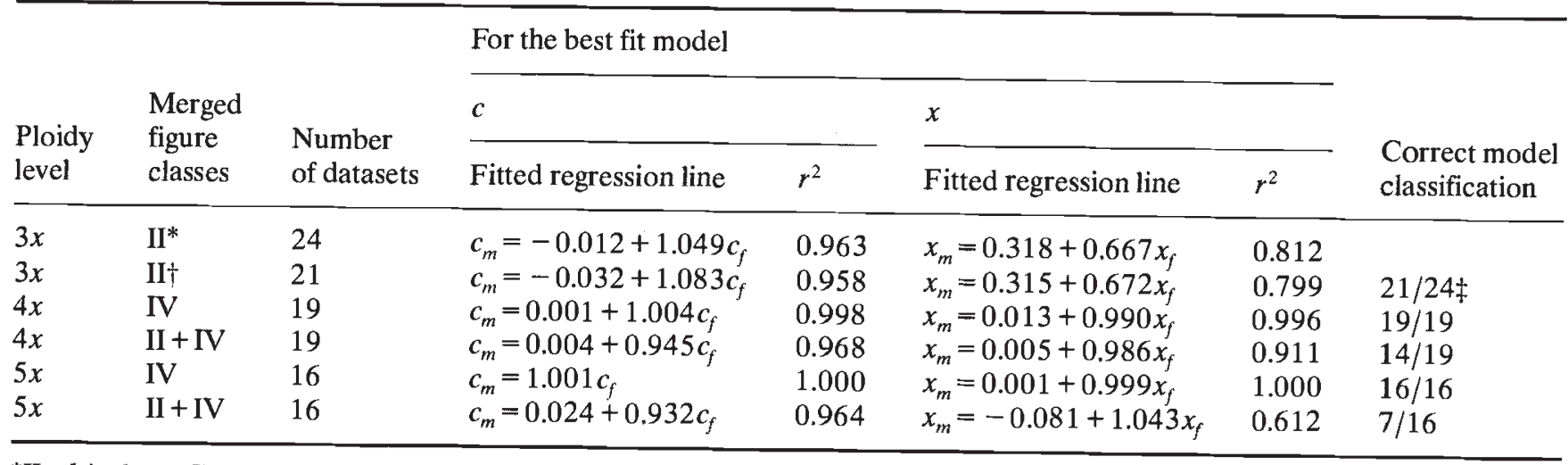

*II = bivalents, IV = quadrivalents.

$\dagger$ Numbers of rod and ring bivalents estimated according to Kimber \& Pignone (1982).

$\ddagger$ In three out of the 24 datasets total bivalents exceeded univalent numbers (see text). 
Table 2 Meiotic analysis of some tetraploid hybrid with merged quadrivalent classes, data from Bothmer et al.(1988)

\begin{tabular}{lrllllllll}
\hline Hybrid & \multicolumn{1}{c}{ I* } & II & III & III & IV & $\begin{array}{l}\text { Best fit } \\
\text { model }\end{array}$ & WSSD & Estimated $c$ & $x$ \\
\hline $\begin{array}{l}\text { Hordeum brevisubulatum } \times \\
\text { H. fuegianum }\end{array}$ & 2.96 & 3.12 & 4.82 & 1.46 & 1.22 & $2: 2$ & 18.912 & 0.719 & 0.891 \\
H. jubatum $\times$ H. feugianum & 10.60 & 4.00 & 3.25 & 0.80 & 0.20 & $2: 1: 1$ & 7.213 & 0.454 & 0.922 \\
H. roshevitzii $\times$ H. jubatum & 0.50 & 3.40 & 7.65 & 0.10 & 1.25 & $2: 2$ & 0.149 & 0.828 & 0.941 \\
H. marinum $\times$ H. secalinum & 10.00 & 2.35 & 2.45 & 2.35 & 0.30 & $3: 1$ & 5.686 & 0.459 & 0.901 \\
\hline
\end{tabular}

*I = univalents; $\amalg I=$ rod bivalents; (II) = ring bivalents; III = trivalents; $I V=$ quadrivalents.

depends largely on the ring bivalent:trivalent ratio, will also be too high.

Another problem arises in the datasets examined at the triploid level. In three cases the total number of bivalents exceeded that of univalents. This situation, arising either from chromosome translocations or nonhomologous pairing, is not allowed by the models used to estimate relative affinity. When the method of Kimber \& Pignone (1982) is applied this leads to an attempt to obtain the square root of a negative number. The method outlined above does not encounter this difficulty but it is fair to question the reliability of the value of $x$ returned on either the modified or original datasets.

The high reliability of the tetraploid and pentaploid models when quadrivalent classes alone are merged indicates that the ratio of chains to rings makes comparatively little contribution to the estimate of $x$. Although in theory quadrivalents can represent up to half of the meiotic figures observed, in practice lower values of $c$ and higher values of $x$ diminish their number and hence the information they represent. The ratio of smaller figures is of far greater importance in establishing a value of $x$ and choosing among the possible models.

Table 2 illustrates the application of this approach to some interspecific Hordeum tetraploid hybrids where the quadrivalent classes were not distinguished. The first hybrid fits the 2:2 model best in agreement with von Bothmer et al.'s (1988) conclusion that $H$. brevisubulatum and $H$. fuegianum share two homologous/ homoeologous genomes, although the fit is poor, which suggests that this explanation is not entirely satisfactory. $H$. jubatum is also thought to carry these same two genomes but its hybrid with $H$. fuegianum fits the $2: 1: 1$ model best. It would seem, therefore, that one of the genomes must have diverged more than the other from its 'primitive' form, a situation reminiscent of the pivotal-differential genome structures recognized in the Triticum/Aegilops genera (Zohary \& Feldman, 1962).

The third hybrid, $H$. roshevitzii $\times H$. jubatum gives a close fit to the $2: 2$ model with a high estimate of $x$ at
0.941. There is discussion in this case as to whether these two taxa in fact constitute one species (Bothmer et al., 1988). A value of $x$ nearer, 1.0, however, might be expected in that case, unless the optimized value found here is too low due to interchange heterozygosity in the hybrid.

The last hybrid fits the $3: 1$ model best with an estimated $c$ value around 0.5 , which indicates that $H$. secalinum has one genome in common with the natural autotetraploid $H$. marinum. Bothmer et al. (1988) reached the same conclusion, suggesting that the genomes of $H$. secalinum must be $\mathrm{H}^{\prime} \mathrm{X}$ as compared with the XX of $H$. marinum.

In 1981 Espinasse and Kimber remarked that 'Many, otherwise excellent, published results' could not be analysed because open and closed figures had not been separately scored. The present paper shows that whilst the problem can be circumvented in some cases, this is no substitute for a full description of the figures observed at metaphase I of meiosis. Indeed attempts to analyse data by the present mathematical techniques can be highly misleading if all classes are not listed. There is no reason to think that subjective assessments would fare any better. Clearly, it is essential that open and closed meiotic figures be recorded separately if the meiotic data are to be used effectively in genomic analysis.

\section{A note on programs}

All the programs for the analysis of meiosis in species hybrids described in this series of papers have been written in TURBo PASCAL to run on the Apple Macintosh. Copies of the source code and the compiled programs are available from the authors upon receipt of an $800 \mathrm{~K}$ or $1.4 \mathrm{MB}$ initialized disk. Use of the source code requires TURBO PASCAL.

\section{Acknowledgements}

CGDC was supported by a post-doctoral fellowship from the University of Missouri. Contribution from the 
Missouri Agricultural Experiment Station. Journal Series Number: 11,260.

\section{References}

ALONSO, L. C. AND KIMBER, G. 1981 The analysis of meiosis in hybrids. II. Triploid hybrids. Can. J. Genet. Cytol., 23, 221-234.

BOTHMER, R., VON, FLINK, J. AND LANDSTROM, T. 1988. Meiosis in interspecific Hordeum hybrids. IV. Tetraploid $(4 x \times 4 x)$ hybrids. Genome, 30, 479-485.

CHAPMAN, C. G. D. AND KIMBER, G. 1992a. Developments in the meiotic analysis of hybrids. I. Review of theory and optimizaton in triploids. Heredity, 68, 97-103.

CHAPMAN, C. G. D. AND KIMBER, G. 1992b. Developments in the meiotic analysis of hybrids. II. Amended models for tetraploids. Heredity, 68, 105-113.
CHAPMAN, C. G. D. AND KIMBER, G. 1992c. Developments in the meiotic analysis of hybrids. III. Amended models for pentaploids. Heredity, 68, 93-200.

ESPINASSE, A. AND KIMBER, G. 1981. The analysis of meiosis in hybrids. IV. Pentaploid hybrids. Can. J. Genet. Cytol., 23, 627-638.

KIMBER, G. AND ALONSO, L. C. 1981. The analysis of meiosis in hybrids. III. Tetraploid hybrids. Can. J. Genet. Cytol., 23, 235-254.

KIMBER, G. AND PIGNONE, D. 1982. The calculation of the frequency of rod and ring bivalents in triploid hybrids. Cereal Res. Commum., 10, 27-31.

ZOHARY, D. AND FELDMAN, M. 1962, Hybridization between amphidiploids and the evolution of polyploids in the wheat (Aegilops-Triticum) group. Evolution, 16, 44-61. 